\title{
Commentary
}

\section{Rhinosinusitis as a cause of headache}

\author{
Diego Belandrino Swerts
}

Albert Einstein Faculty of Medicine, Neurology, São Paulo, São Paulo, Brazil

\begin{abstract}
$\triangle$ The article published by Ceriani and Silberstein' addresses, in the format of a systematic review, the diegobelandrino@gmail.com relationship between headache and rhinosinusitis, showing that most of these cases are over diagnosed in patients who actually have primary headaches, the majority being migraines. In addition, the article emphasizes the underdiagnosed sphenoid rhinosinusitis which can have great morbidity and even mortality.
\end{abstract}

The article reviews the concepts of acute rhinosinusitis (1 day to 4 weeks), recurrent acute respiratory syndrome (ARS) (four or more episodes lasting 7 days in one year) and chronic rhinosinusitis (persistent beyond 12 weeks). The article then discusses the morphological, etiological, diagnostic and treatment review of these diseases.

Acute respiratory syndromes are mostly viral, with adenovirus, influenza and parainfluenza as the most prevalent agents. Bacterial infections have as main agents Streptococcus pneumoniae and Haemophilus influenza. The clinical presentation usually leads to purulent nasal discharge, accompanied by nasal obstruction and/or pain pressure fullness. Altered sense of smell, cough, halitosis, dental pain, pharyngitis, and otologic symptoms may be present.

Migraine symptoms, such as nausea, photophobia, phonophobia were seen in the minority of patients in the studies. In addition, ARS headache is bilateral, in pressure with worsening frontal compression, less common symptoms in primary headaches. In chronic respiratory syndrome (CRS), headache and facial pain are even less common symptoms. The studies analyzed in the systematic review show that primary headaches should always be considered in patients with rhinosinusitis, and most patients in the studies ended up switching the diagnosis to primary headache after revaluation.

The article continues with a review of treatment of ARS cases, showing that symptomatic patients should be the first option, in cases with suspected bacterial agents, amoxicillin proved to be the first-line treatment, with association with clavulanate in patients with suspected resistance to amoxicillin (smokers, diabetics, recent use of antibiotics), the treatment of CRS was indicated with topical corticosteroids and saline wash.

Finally, the article discusses the importance of suspecting sphenoid rhinosinusitis, which can develop with photophobia, lacrimation, cranial nerve palsies, fever, epistasis, and visual symptoms. The importance of investigating for this condition is due to complications such as bacterial meningitis, sinus thrombosis, abscess, hypophysitis that are associated with high morbidity and even mortality.

Thus, in a very well-written article, the authors go through the management of the patient with a previous diagnosis of rhinosinusitis, reporting through the symptoms, the importance of reviewing the diagnosis and suspecting primary headaches. In addition, it eases the management of these conditions, and emphasizes the importance of excluding the diagnosis of sphenoidal rhinosinusitis in these cases.

\section{References}

Ceriani CE, Silberstein SD. Headache and rhinosinusitis: A review. Cephalalgia 2021;41:453-463 